\title{
ResearchOnline@JCU
}

This is the author-created version of the following work:

O'Brien, Freya, and Thorley, Craig (2021) Memory of people from missing person posters: the number of posters seen, the number of times they are seen, and the passage of time matter. Psychology, Crime \& Law, 27 (8) pp. 779-795.

Access to this file is available from:

https://researchonline.jcu.edu.au/64575/

Published Version: (C) 2020 Informa UK Limited, trading as Taylor \& Francis Group. Accepted Version may be made open access without embargo.

Please refer to the original source for the final version of this work: 
Memory of people from missing person posters: The number of posters seen, the number of times they are seen, and the passage of time matter

\author{
Freya O’Brien ${ }^{1}$ \\ Craig Thorley ${ }^{2}$ \\ ${ }^{1}$ School of Psychology, University of Liverpool \\ ${ }^{2}$ Department of Psychology, James Cook University
}

Author Notes:

Correspondence concerning this article should be addressed to Dr. Freya O'Brien,

School of Psychology, Eleanor Rathbone Building, University of Liverpool, England, L69 7ZA. Email: F.Obrien@liverpool.ac.uk 


\begin{abstract}
Missing person posters ask the public to monitor their environment for missing people and alert the authorities if they are encountered. Here, a single laboratory-based study examined whether the number of missing person posters someone sees, the number of times the posters are seen, and the time delay between seeing the posters and later encountering the missing people from them all influence the likelihood of the missing people being recognised and authorities alerted. To examine this, participants saw four or 12 missing person posters once, three, or five times and were asked to alert the researchers if any missing people later appeared in the study. Shortly after, or after a minimum of a 24-hr delay, the participants had to sort pictures of 40 people by gender. Four of these 40 people had appeared on the missing person posters. Participants were more likely to recognise the missing people and alert the researchers of this when they had seen four posters three or five times and when the missing people were encountered shortly after seeing the posters. When the posters were seen once, however, the number of posters seen did not impact performance. The applied implications of these findings are discussed.
\end{abstract}

Keywords: missing people, memory, posters, prospective memory, time delay 


\section{Memory of people from missing person posters: The number of posters seen, the number of times they are seen, and the passage of time matter}

In 2019, law enforcement agencies in the United States created 609,275 new missing person records (National Crime Information Centre, 2020). When someone goes missing, the general public can be asked to help locate them. This request may be made via missing person posters placed in high foot-traffic areas such as supermarkets and train stations. The posters typically contain the missing person's image, name, age, and a telephone number to call to alert authorities if the missing person is sighted. It is hoped that members of the public who see the poster and then encounter the missing person will recognise the missing person and alert authorities of the sighting. The research reported in this article examined whether the number of missing person posters a person sees, the number of times the posters are seen, and the time delay between seeing the posters and encountering the missing people from them all influence the likelihood of the missing people being recognised and others alerted.

\section{Prospective Person Memory}

Lampinen and Moore (2016a) suggest that if a member of public is to use a missing person poster to help locate a missing person, several things must happen. First, the member of public must encounter the missing person poster. They must then attend to the poster and memorise what the missing person looks like. Afterwards, they must encounter the missing person, attend to the missing person, recognise the missing person, and remember to alert the authorities that the person was sighted. The member of public must therefore utilise several cognitive processes, including attention, face recognition, and event-based prospective memory (Lampinen \& Moore, 2016a, Moore \& Lampinen, 2019). Event-based prospective memory involves remembering to perform an intended action upon encountering a cue (Einstein \& McDaniel, 1990). In the context of the present study, the cue is a missing person and the action involves alerting the authorities. When a member of public attends to a missing 
person poster, later encounters and recognises the missing person, and then remembers to alert the authorities, they are said to have utilised Prospective Person Memory or PPM (Lampinen, Arnal, \& Hicks, 2009, Lampinen \& Moore, 2016a).

PPM studies try to simulate the search for a missing person. They can be conducted using either laboratory or field methods. In laboratory studies, participants typically study several mock missing person posters, or similar appeals, and are asked to alert the researchers if any of the missing people are later encountered during the study. The participants then complete a task, such as sorting images of strangers into groups, and several of the missing people appear during that task (e.g., Lampinen, Arnal, Adams, Courtney \& Hicks, 2012a; Lampinen, Peters, \& Gier, 2012b; Sweeney \& Lampinen, 2012). In field studies, participants typically study one mock missing person poster, or a similar appeal, and are asked to alert the researchers via email if the missing person is later encountered in their everyday lives. Over subsequent days, the missing person then appears at locations the participants frequent (e.g., Lampinen, Curry, \& Erickson, 2016; Lampinen \& Moore, 2016b; Moore, Lampinen, \& Provenzano, 2016). In both types of study, PPM performance is measured by calculating how often participants correctly recognise a missing person/remember to alert the researchers of this encounter. PPM performance is superior in laboratory studies. For example, in Lampinen et al.'s (2012b, Experiment 1) laboratory study, the success rate was 56.5\% but in Lampinen and Moore's (2016b) field study it was close to 7\%. These different success rates are unsurprising as laboratory studies ensure participants attend to a missing person upon encountering them but field studies do not.

Laboratory and field studies have identified several factors that influence PPM performance (see Lampinen \& Moore, 2016a, for a review). To give some examples, PPM performance is superior when posters contain more than one image of a missing person (Sweeney \& Lampinen, 2012) and when there is a match between a missing person's 
appearance on a poster and their appearance when later encountered (e.g., Gier \& Kreiner, \& Hudnell, 2012). Three other factors that may influence PPM performance are the number of missing person posters a person sees, the number of times the posters are seen, and the time delay between seeing the posters and encountering a missing person from them.

\section{Number of Posters Seen}

Large numbers of missing person posters can sometimes be displayed together in high foot-traffic areas such as supermarkets and train stations. For example, Lampinen et al. (2012b) point out that missing child poster boards in Walmart stores contain space for up to 15 posters. Lampinen et al. (2012b) questioned whether presenting members of the public with a large number of posters is sensible as they may struggle to remember each poster, relative to if they only saw a small number of posters, reducing the likelihood of PPM occurring if a missing person is later encountered. To investigate this, Lampinen et al. (2012b, Experiment 1) ran a laboratory study where adult participants studied four or 12 mock missing child posters on a computer screen for $15 \mathrm{sec}$ each. The participants were told that if they later encountered any of the missing children in the study, they should 'alert the authorities' via a keyboard button press. Afterwards, the participants had to sort 46 pictures of children into two teams. Four of the 46 children were missing children and PPM performance was measured. Lampinen et al. (2012b, Experiment 1) found that the number of posters participants saw did not impact upon PPM performance ${ }^{1}$.

Lampinen et al.'s (2012b, Experiment 1) demonstration that PPM performance is similar when participants study four or 12 missing person posters is perhaps unsurprising when considered alongside findings from the broader face recognition literature. In that

\footnotetext{
${ }^{1}$ Lampinen et al. (2012b, Experiment 2) also ran a second study where participants spent more time studying each of the four posters, relative to each of the 12 posters. In that study, PPM performance was best after studying four posters. Thus, PPM declined as the amount of time spent studying each individual poster was reduced. This likely occurred as reduced exposure times to faces creates weaker memories of them (Memon, Hope, \& Bull, 2003).
} 
literature, it has been shown that participants can study 20 faces in one session for $5 \mathrm{sec}$ each and later correctly recognise them with a high degree of accuracy (Podd, 1990; see also Chance \& Goldstein, 1979; Goldstein \& Chance, 1970). It is therefore possible that it was no more difficult to recognise 12 recently studied faces than four (but see a meta-analysis by Shapiro \& Penrod, 1986, for a discussion of the conditions under which studying a large number of faces may impair later recognition). Lampinen et al.’s (2012b, Experiment 1) finding is, however, perhaps surprising when considered alongside findings from the broader prospective memory literature. A recent meta-analysis by Anderson, Strube, and McDaniel (2019) showed a small-to-medium sized negative relationship $(r=-.18)$ between the number of event-based prospective memory cues a person must remember and the likelihood of them prospectively remembering to carry out the act associated with the cues when they are next encountered. For example, in one of the meta-analysis studies, Cohen (2013) found that as the number of cues that needed remembering increased from one to six, the percentage of times the act associated with the cues was carried out when they were next encountered declined from $83 \%$ to $58 \%$. One shortcoming of Lampinen et al.'s (2012b, Experiment 1) study was that their sample size was too small to detect small-to-medium sized effects. Given that their finding runs counter to what would be predicted from the prospective memory literature, it would be beneficial to try and replicate their null finding with a larger sample size in case a small or medium sized effect was missed.

Finally, Lampinen et al. (2012b, Experiment 1) also examined whether participants had any response bias during the team sorting task. A liberal response bias would occur if participants had a tendency to class children as missing, regardless of whether they were missing or not. A conservative response bias would occur if participants had a tendency to class children as not missing. A conservative response bias was observed but it was reduced when participants had studied 12 posters. Whilst the reasons for this were not established, 
two potential explanations were offered. First, exposing participants to a greater number of posters could have increased the probability of some making a spurious facial match between the non-missing and missing children, meaning they mistook the former for the latter. Second, participants who studied 12 posters may have expected more than four missing children to appear during the team sorting task and been more liberal as a result of this.

\section{Repeated Exposure to Posters and The Passage of Time}

No previous studies have examined whether the number of times a participant sees a missing person poster in a short space of time can influence PPM performance. If repeated exposures are beneficial, this could potentially inform best practice when disseminating posters in public. For example, if repeated exposure to a poster improves PPM performance, it may be best to place multiple copies of the poster in nearby geographical locations (e.g., on a supermarket missing person's poster board and at locations nearby) instead of distant geographical locations (e.g., on separate supermarket missing person's poster boards) as doing so could increase the number of times a member of the public is exposed to the poster in a short space of time. There is indirect evidence to suggest that repeated exposure to a missing person poster within a short space of time may improve PPM performance. For example, repeated exposure to faces in a short space of time can improve later recognition of them (e.g., Feng et al., 2019; Roark, O'Toole, Abdi, \& Barrett, 2006; Russo, Parkin, Taylor, \& Wilks, 2008; Xue et al., 2011). Similarly, repeated exposure to a prospective memory cue increases the likelihood of remembering to carry out the intention associated with it when the cue is next encountered (Guynn \& McDaniel, 2007).

It is also the case that no previous studies have examined whether the time delay between seeing a missing person poster and encountering a missing person influences PPM performance. If delays are harmful, this could also potentially inform best practice when disseminating posters in public. For example, if a missing person on a once-seen poster is 
likely to be quickly forgotten, consideration can be given as to how to prevent this (e.g., by increasing opportunities for re-exposure to the poster). There is indirect evidence to suggest the passage of time may impact PPM. For example, it has been shown that the rate at which once-seen unfamiliar faces are forgotten follows an Ebbinghausian-type forgetting curve, with forgetting being greatest shortly after seeing the faces and then levelling off (see Deffenbacher, Bornstein, McGorty, \& Penrod, 2008, for a meta-analysis). Additionally, when the delay between forming a prospective memory intention and encountering the cue to act it out is longer than a few hr, this can impair prospective memory performance (see Martin, Brown, \& Hicks, 2011, for a review and exceptions).

\section{Aims and Hypotheses}

Our study had three aims. The first aim was to try and replicate Lampinen et al.'s (2012b, Experiment 1) finding that the number of missing person posters participants see does not influence PPM performance. As in their study, participants studied four or 12 missing person posters and then completed a sorting task which included four of the missing people. One difference between the two studies is that our missing and non-missing people were adults, not children. Another difference is that our study had a high-powered analysis capable of detecting small effects. The second aim was to examine whether the number of times missing person posters are seen influences PPM performance. To determine this, participants saw the posters once, three, or five times. The third aim was to examine whether the passage of time between seeing missing person posters and encountering the missing people from them influences PPM performance. To determine this, participants completed the sorting task shortly after seeing the missing person posters or after a minimum of a 24-hr delay. As in Lampinen et al. (2012b, Experiment 1), response bias was also analysed.

It was possible to make several predictions. First, it was predicted that the number of missing person posters participants studied would not impact upon their PPM performance. 
This was based on a similar finding by Lampinen et al. (2012b, Experiment 1) and research showing participants can study more than 12 faces once, for a few sec each, and later recognise them with a high degree of accuracy (e.g., Podd, 1990). As mentioned however, a recent meta-analysis found a small-to-medium sized negative relationship between the number of event-based prospective memory cues that need storing in memory and prospective memory performance (Anderson et al. 2019) and Lampinen et al.'s analysis was not sufficiently powered to detect small-to-medium sized effects. We could not, therefore, rule out the possibility that studying a greater number of posters would impair PPM performance. In line with Lampinen et al. (2012b, Experiment 1), it was also predicted that participants would have a conservative response bias but that this would be reduced when they saw 12 posters. It was also predicted that repeated exposure to the missing person posters would improve PPM performance. This prediction was based upon past research showing repeated exposure to faces improves recognition of them (e.g., Roark et al., 2006), whilst more than one exposure to a prospective memory cue improves prospective memory performance (Guynn \& McDaniel, 2007). It was also predicted that PPM performance would decline when there was more than a 24-hr delay between seeing the posters and encountering the missing people. This latter prediction was based upon past research showing memory of faces degrades over time (e.g., Deffenbacher et al., 2008) and that long delays between forming a prospective memory intention and encountering the cue to act it out can reduce prospective memory performance (see Martin et al., 2011). Due to a lack of past research, analyses examining the impact of the number of exposures and time delay on response bias were exploratory. 


\section{Method}

\section{Participants}

There were 574 participants ( 487 females, 85 males, 2 other). They were aged 17 - 77 $(M=23.20, S D=11.41)$. Of these, 351 were students studying introductory psychology classes who participated for course credit. The remainder were non-psychology students, university staff, and members of the public who responded to e-advertisements for participants and volunteered their time. All provided informed consent prior to participation. The study took place online.

This sample size has enough power $(\beta=.80)$ to detect small effects (Cohen's $f=.13)$ in our main $2 \times 3 \times 2$ between-subjects ANOVA analyses when $\alpha$ is .05 (G*Power 3; Faul, Erdfelder, Lang, \& Buchner, 2007). It was important the analysis could detect small effects as one aim of this study was to examine whether the number of missing person posters participants see, and therefore the number of missing people they must remember, impacts upon PPM performance. As mentioned, a recent meta-analysis showed there is a small-tomedium sized negative relationship between the number of prospective memory cues a person must remember and prospective memory performance (Anderson et al., 2019). In our study, the missing people are the prospective memory cues. Thus, if our analysis could not detect potentially small effects, a Type 2 Error could occur.

\section{Design}

There were three between-subjects independent variables. These were the number of missing person posters participants saw (four or 12), the number of times they saw each poster (one, three, or five), and the time delay between seeing the posters and completing the sorting task in which they were re-exposed to four people from the missing person posters (no time delay or a minimum of a $24 \mathrm{hr}$ delay). 
There were two dependent variables. These were PPM performance and response bias. They are equivalent to discriminability and response bias in Signal Detection Theory. Further details regarding their calculation and interpretation are provided in the Results section. In brief, PPM performance/discriminability is a single measure of a participant's ability to discriminate missing and non-missing people during the sorting task and correctly alert the researchers that a missing person has been encountered. Response bias is a measure of a participant's response strategy. If a participant had a liberal response bias, they would tend to identify people as missing during the sorting task regardless of whether they were missing or not. If a participant had a conservative response bias, they would tend to identify people as not missing during the sorting task regardless of whether they were missing or not.

All participants were randomly allocated to their conditions. Due to non-completers, each condition had different sample sizes (see Table 1). Non-completers are people who clicked on the link to the study, accessing the information sheet, but did not commence the study proper or people who commenced the study proper but did not finish it. The Supplemental Materials has a breakdown of how many non-completers there were in each condition. The overall attrition rate $(15.84 \%)$ was lower than is typically found in online studies, where rates between $30 \%$ and 50\% are common (see Zhou \& Fishbach, 2016).

\section{Materials}

There were 12 missing person posters (see Figure 1 for an example). The posters were modelled on those used by Missing People, which is a missing person's charity in the country where this study was conducted. Each poster had one photograph of an allegedly missing person. The people depicted as missing were amateur actors whose photographs were taken from The Karolinska Directed Emotional Faces database (KDEF; Lundqvist, Flykt \& Öhmen, 1998). All 'missing people' were White, aged 20 - 30, and there was an even male/female gender split. Photographs of young White adults were chosen as they are most likely to go 
missing in the country where this study was conducted (National Crime Agency, 2019). In all photographs, the missing person was looking straight at the camera, had a neutral facial expression, and was shown from the neck up. Beneath each photograph was the missing person's name, created using a random name generator, and a fictional helpline number. When participants saw a missing person poster more than once, they saw the exact same poster each time. Four posters were target posters, with the missing people ( 2 males, 2 females) also appearing during the sorting task. Eight posters were distractor posters, with the missing people not appearing during the sorting task.

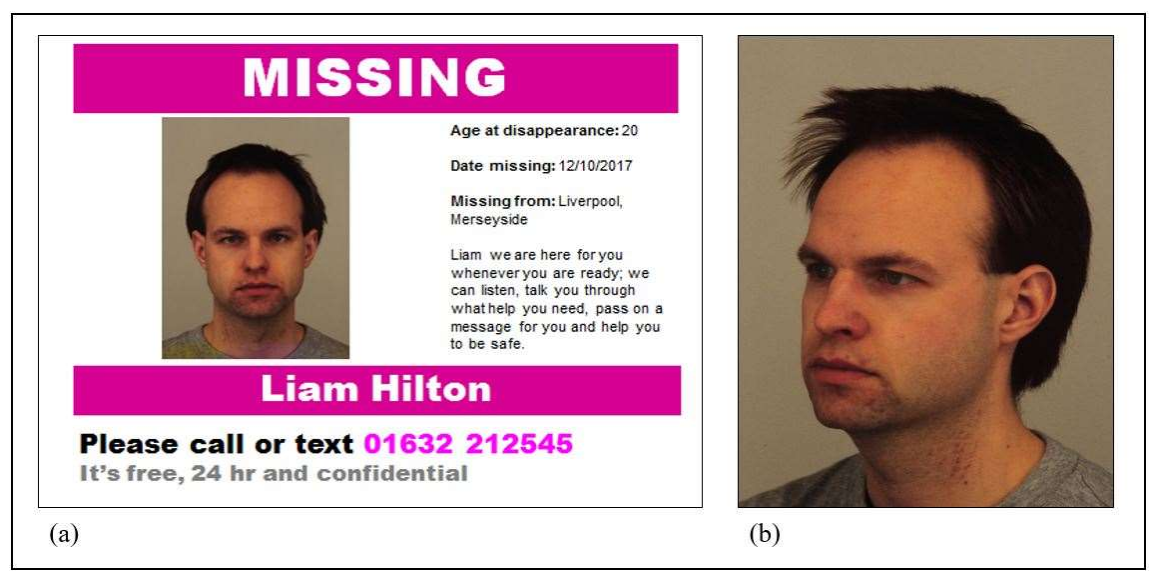

Figure 1. Image (a) shows a missing person poster used in this study and image (b) shows the alternative photograph of the missing person used in the sorting task. Both images are from the KDEF database, with (a) being image AM02NES and (b) being image AM02NEHR

The sorting task contained photographs of 40 amateur actors from the KDEF database. As mentioned, four had been depicted as missing people on the target posters. During the sorting task, alternative photographs of these same four missing people were used. In these alternative photographs, they appeared in a three-quarter profile pose but still had a neutral facial expression and were shown from the neck upwards (see Figure 1 for an example). The remaining 36 people were new. These new people also appeared in a threequarter profile pose, had a neutral facial expression, were shown from the neck up, and were matched with the missing people in terms of their ethnicity, age range, and gender split. 


\section{Procedure}

In line with Lampinen et al. (2012b), participants were directed to a web page where they were told they would be participating in a study mimicking the search for missing people. They were then told that they would be required to study several missing person posters and that some may be seen more than once. Importantly, they were told that if they saw any of the missing people in subsequent parts of the study, they should type 'missing' into the computer to alert the researchers of the sighting. The participants then saw four or 12 missing person posters, with the posters presented separately in a random order for $5 \mathrm{sec}$ each. Some participants saw each poster three or five times. When this happened, the posters were presented in blocks. Each block contained one copy of each poster. For example, if participants were exposed to 12 posters and saw each five times, they first saw all 12 posters (one at a time) in a random order, then saw all 12 again (one at a time) in a random order, etc.

After studying the missing person posters, the participants were randomly assigned to complete the sorting task either immediately or after a $24-\mathrm{hr}$ delay. Those in the delay condition were automatically emailed the link to the sorting task $24 \mathrm{hr}$ later and were asked to complete it as soon as possible thereafter. No deadline for completing it was enforced. Prior to the sorting task, participants were instructed that they would see 40 photographs of strangers and they had to sort the photographs by gender, typing 'male' into the computer for males and 'female' into the computer for females. They were also told that they should type 'missing' into the computer instead of the person's gender if they saw any of the people from the missing person posters. They then commenced the sorting task, with each photograph shown separately. Once participants had responded to a photograph, the next photograph appeared. Participants could not change earlier responses. The sorting task was self-paced. Afterwards, the study ended. 


\section{Results}

The data that support the findings of this study are available from the corresponding author upon reasonable request.

\section{Preliminary Analysis}

Prior to analysing each groups' PPM performance and response bias, it was important to know whether the six delay group conditions experienced an equivalent delay between seeing the missing person posters and completing the sorting task. If some groups experienced a longer delay than others, this would create a confound in the study. The Mean delay across all six conditions was $41.29 \mathrm{hr}(M d n=28.08 \mathrm{hr}, S D=33.66 \mathrm{hr}$, range $=37.98 \mathrm{hr}$ $-48.52 \mathrm{hr}$ ). The delay data was positively skewed in each condition and a Kruskal-Wallis test showed the delays across each condition did not significantly differ, $H(5)=10.74, p=.06$.

\section{Signal Detection Theory Analysis Overview}

As mentioned, our PPM performance and response bias measures are equivalent to discriminability and response bias measures in Signal Detection Theory (SDT), respectively. In SDT, both measures are calculated using the proportion of hits and false alarms made by participants during recognition tasks. In the present study, a hit occurred when a participant correctly recognised a missing person during the sorting task and alerted the researchers of the encounter. A false alarm occurred when a participant incorrectly recognised a nonmissing person as a missing person during the sorting task and mistakenly alerted the researchers a missing person had been encountered. Interested readers should see Stanislaw and Todorov (1999) for a detailed overview of SDT and the calculations used to generate discriminability and response bias measures from hits and false alarms. Discriminability and response bias measures exist for both parametric analyses (called $d^{\prime}$ and $C$, respectively) and non-parametric analyses (called $A^{\prime}$ and $B^{\prime \prime}$, respectively). 
In the current study, Shapiro-Wilk tests $(\alpha=.05)$ and Normal Q-Q Plots showed participants' PPM performance/discriminability and response bias data were non-normally distributed in several conditions, meaning non-parametric SDT measures were required for all analyses. With these non-parametric measures, $A^{\prime}$ typically ranges from .5 (indicating missing people cannot be distinguished from non-missing people) to 1 (indicating perfect discrimination). Values less than .5 may arise from sampling error or response confusion; 0 is the lowest possible value. $B^{\prime \prime}$ ranges from -1 (an extreme liberal response bias) to 1 (an extreme conservative response bias). A value of 0 signifies no response bias. The Mean hits, false alarms, $A^{\prime}$ scores, $B^{\prime \prime}$ scores per condition, and associated standard deviations, are in Table 1. An $\alpha$ of .05 was used as the cut-off point for statistical significance in all analyses.

\section{$A^{\prime}$ Analysis (PPM Performance)}

A $2 \times 3 \times 2$ between-subjects ANOVA was used to examined the impact of the number of missing person posters seen (four or 12 posters), the number of times they were seen $(1,3$, or 5$)$, and the delay between seeing them and completing the sorting task (no delay or $24+\mathrm{hr}$ ) on PPM performance using $A^{\prime}$ (or discriminability) as the dependent measure. Omega-squared $\left(\omega^{2}\right)$ was used as an effect size measure with values of $0.01,0.06$, and 0.14 indicating small, medium, and large effect sizes respectively.

The main effect for the number of posters seen was statistically significant, $F(1,562)$ $=11.50, \mathrm{MSe}=.05, p<.001, \omega^{2}=.02$, with PPM performance best when participants saw four posters $(M=.71, S D=.23)$ relative to $12(M=.65, S D=.23)$. The main effect for the number of times posters were seen was also statistically significant, $F(2,562)=19.55, M S e=$ $.05, p<.001, \omega^{2}=.06$. Planned comparisons, with observed $\mathrm{p}$-values multiplied by three to control for Type 1 Errors, showed PPM performance was superior when participants saw the missing person posters three times $(M=.72, S D=.21)$ or five times $(M=.71, S D=.23)$ relative to once $(M=.59, S D=.23$, both $p$ 's $<.001)$. PPM performance did not significantly 
differ after seeing the posters three or five times $(p=.93)$. Finally, the main effect of delay was also statistically significant, $F(1,562)=4.56, M S e=.05, p=.03, \omega^{2}=.001$, with PPM performance superior when the sorting task was completed without a delay $(M=.70, S D=$ $.23)$ relative to a $24+\mathrm{hr}$ delay $(M=.67, S D=.23)$. No interactions were statistically significant (all $p$ 's $>.11)$.

To summarise the findings, participants were more likely to correctly recognise missing people and alert the researchers of this during the sorting task when they saw four missing person posters (relative to 12), saw the posters three or five times (relative to once), and when they completed the sorting task shortly after seeing the posters (relative to after a $24+\mathrm{hr}$ delay). The first and last of these effects were small, but the effect of the number of times posters were seen was medium sized.

One aim of the present study was to try and replicate Lampinen et al.'s (2012b, Experiment 1) finding that PPM performance is equivalent when participants see four or 12 missing person posters. As mentioned, our ANOVA analysis revealed a main effect of the number of posters seen, whereby PPM performance was best when participants saw four posters. An examination of the $A^{\prime}$ values in Table 1 suggests this main effect was partially driven by performance improvements when participants saw four posters three or five times and the sorting task occurred without a delay. Importantly, the conditions in our study that most closely match Lampinen et al.'s (2012b, Experiment 1) are those where participants saw four or 12 posters once and the sorting task occurred without a delay. In the present study, PPM performance in those two conditions was similar, with the $A^{\prime}$ values being .64 and .61 respectively. An independent samples t-test showed they did not significantly differ, $t(99)=$ $0.75, p=.46, d=.15$. Thus, our study replicated Lampinen et al.'s (2012b, Experiment 1) finding that PPM performance is equivalent when participants see four or 12 posters, provided the posters are seen once and the missing people are encountered shortly after. 
Table 1

The proportion of hits and false alarms, and the Mean $A^{\prime}$ (PPM performance) and $B^{\prime \prime}$ (response bias) scores during a test that required identifying four people from previously studied missing person posters. Participants initially studied four or 12 missing person posters once, three times, or five times and were re-exposed to four missing people from the posters either immediately or after a 24-hr delay

\begin{tabular}{|c|c|c|c|c|c|c|}
\hline $\begin{array}{l}\text { Number of } \\
\text { Posters Studied }\end{array}$ & $\begin{array}{l}\text { Number of } \\
\text { Exposures }\end{array}$ & $n$ & Hits & $\begin{array}{l}\text { False } \\
\text { Alarms }\end{array}$ & $A^{\prime}$ & $B^{\prime \prime}$ \\
\hline & & & & $\underline{\text { No Dela }}$ & & \\
\hline \multirow[t]{3}{*}{ Four } & One & 51 & $.42(.31)$ & $.18(.11)$ & $.64(.25)$ & $.26(.43)$ \\
\hline & Three & 62 & $.56(.29)$ & $.14(.11)$ & $.77(.20)$ & $.24(.48)$ \\
\hline & Five & 48 & $.56(.27)$ & $.12(.12)$ & $.78(.22)$ & $.39(.48)$ \\
\hline \multirow[t]{4}{*}{ Twelve } & One & 50 & $.44(.30)$ & $.27(.16)$ & $.61(.21)$ & $.06(.40)$ \\
\hline & Three & 51 & $.50(.31)$ & $.20(.12)$ & $.69(.22)$ & $.12(.45)$ \\
\hline & Five & 46 & $.48(.34)$ & $.21(.14)$ & $.67(.24)$ & $.13(.43)$ \\
\hline & & \multicolumn{5}{|c|}{ Delay } \\
\hline \multirow[t]{3}{*}{ Four } & One & 30 & $.33(.25)$ & $.16(.11)$ & $.61(.20)$ & $.32(.30)$ \\
\hline & Three & 65 & $.43(.32)$ & $.12(.12)$ & $.70(.22)$ & $.29(.45)$ \\
\hline & Five & 44 & $.47(.31)$ & $.12(.11)$ & $.73(.22)$ & $.31(.45)$ \\
\hline \multirow[t]{3}{*}{ Twelve } & One & 37 & $.28(.26)$ & $.25(.14)$ & $.49(.23)$ & $.36(.38)$ \\
\hline & Three & 53 & $.54(.28)$ & $.20(.12)$ & $.72(.20)$ & $.12(.41)$ \\
\hline & Five & 37 & $.45(.29)$ & $.18(.14)$ & $.67(.22)$ & $.26(.37)$ \\
\hline
\end{tabular}

\section{$B^{\prime \prime}$ Analysis (Response Bias)}

The Mean $B^{\prime \prime}$ scores across all conditions were positive (grand $M=.23, S D=.44$ ), meaning participants had a bias towards classing people as not missing during the sorting task. A $2 \times 3 \times 2$ between-subjects ANOVA was used to examine whether participants' response bias differed as a result of the number of missing person posters seen, the number of times the posters were seen, and the time delay between seeing the posters and completing the sorting task. There was a main effect of the number of posters seen, $F(1,562)=11.73$, $M S e=.18, p<.001, \omega^{2}=.02$, with participants more likely to class people as not missing after seeing four posters $(M=.30, S D=.45)$ relative to 12 posters $(M=.16, S D=.42)$. There 
was no main effect of the number of times posters were seen, $F(2,562)=1.86, M S e=.18, p$ $=.16, \omega^{2}=.003$. There was, however, a main effect of delay, $F(1,562)=4.24, M S e=.18, p$ $=.04, \omega^{2}=.005$, with participants more likely to class people as not missing when there was a $24+\mathrm{hr}$ delay between seeing the posters and completing the sorting task $(M=.27, S D=$ $.41)$, relative to when there was no delay $(M=.20, S D=.46)$. There were no statistically significant interactions (all p's >.08).

To summarise the findings, participants had a bias towards classing people as not missing and this bias was reduced by a small, but statistically significant, amount after seeing 12 missing person posters (relative to four) and when completing the sorting task shortly after seeing the posters (relative to after a $24+\mathrm{hr}$ delay).

\section{Discussion}

This study examined whether the number of missing person posters a person sees, the number of times the posters are seen, and the delay between seeing the posters and later encountering the missing people from them can all impact upon their PPM performance. To assess this, participants studied four or 12 missing person posters once, three times, or five times and then, either immediately or after a $24+$ hour delay, encountered four of the missing people and 36 non-missing people. PPM performance was assessed by examining whether participants correctly recognised the missing people and remembered to alert the researchers a missing person had been encountered. PPM performance was best after seeing four posters three or five times and when the missing people were encountered shortly after seeing the posters. When posters were seen once, however, the number seen did not impact upon PPM performance. Additionally, participants had a bias towards classing people as not missing, regardless of whether they were or not, but this was reduced slightly when they had seen 12 posters and encountered the missing and non-missing people shortly after seeing the posters. Each finding is discussed in more detail next. 


\section{Number of Posters Seen}

Previously, only Lampinen et al. (2012b, Experiment 1) had examined whether the number of missing person posters a participant sees influences PPM performance. They had adult participants study four or 12 missing children posters, with each poster seen once for 15 sec, and there was no delay between seeing the posters and later encountering the missing people. They found the number of posters seen did not influence PPM performance. In the present study, however, there was a small overall effect of the number of posters seen on PPM performance, with performance being slightly better when participants saw four posters. Our findings therefore seemingly differ to those of Lampinen et al. (2012b, Experiment 1). Closer inspection of our findings suggests this effect was partially driven by performance improvements when participants saw four posters three or five times and the missing people were encountered shortly after seeing the posters. When a separate analysis was conducted focussing on the conditions most like those in Lampinen et al.'s (2012b, Experiment 1) study, their null effect was replicated. Thus, when participants study four or 12 missing person posters once, each poster is seen for an equal amount of time, and the missing people are encountered shortly afterwards, the number of posters seen does not seemingly impact upon PPM performance. However, seeing a small number of posters several times, as opposed to a large number of posters several times, appears to aid PPM performance if each poster is seen for an equivalent amount of time and the missing people are encountered shortly after. Importantly, the present study extends Lampinen et al.'s (2012b, Experiment 1) finding to a new population of missing people, as theirs were children and ours were young adults.

It is important to consider why Lampinen et al. (2012b, Experiment 1) and the present study both found the number of missing person posters participants see does not impact upon PPM performance, provided each poster is seen once. Readers may find these null effects surprising given that a recent meta-analysis in the broader event-based prospective memory 
literature found evidence of a small negative relationship between the number of cues participants must remember and the likelihood of them remembering to carry out an intention associated with those cues when they are next encountered (Anderson et al., 2019). In PPM studies, the 'cues' are the missing people and the 'intention' is alerting the researchers a missing person has been encountered. With the findings of the recent meta-analysis in mind, it is reasonable to expect that PPM performance would be worse when participants had to remember 12 missing people, relative to four. The present study's analysis had enough statistical power to detect small effects, so it is unlikely a Type 2 Error occurred.

It is likely both Lampinen et al. (2012b, Experiment 1) and the present study found the number of missing person posters participants see, when the posters are seen once, does not impact upon PPM as the PPM tasks they used were not true event-based prospective memory tasks (see Einstein \& McDaniel, 2005, or McDaniel \& Einstein, 2007, for overviews of what constitutes a prospective memory task). In true event-based prospective memory tasks, participants are asked to remember one or more cues and then form an intention to perform an action if the cue(s) are later encountered. The participants then complete a filler task so that the intention leaves their working memory. Finally, the participants complete an ongoing task that has the cue(s) embedded in it. Importantly, participants are not reminded of the intention prior to the ongoing task. Thus, when they encounter the cue(s) during the ongoing task, the participants must retrieve the intention from long-term memory. The retrieval of the intention from long-term memory makes the task a true prospective memory task. Here, and in Lampinen et al.'s (2012b, Experiment 1) study, participants were reminded of the intention immediately before the ongoing task (i.e., the sorting task), so the intention was likely in their working memory during that task and they did not need to retrieve it from long term memory when a missing person was encountered. If so, both studies would be more akin to face recognition studies than true event-based prospective memory studies. 
When this is considered, the null effects they observed are less surprising. Face recognition research shows that participants can study 12 faces in one session and later correctly recognise them with a high degree of accuracy (Chance \& Goldstein, 1979; Goldstein \& Chance, 1970; Podd, 1990). It may, therefore, have been no more difficult to recognise 12 recently studied faces than four recently studied faces in our study and Lampinen et al.'s (2012b, Experiment 1). It would be interesting to know whether the null effects observed in both studies would persist if participants were not reminded of the intention immediately prior to the sorting task, so the studies provided a purer test of event-based prospective memory.

Finally, our study replicated Lampinen et al.'s (2012b, Experiment 1) observation that participants' conservative response bias is reduced after seeing 12 posters, meaning they are less likely to class people as not missing. This effect was small. Lampinen et al. (2012b) offer some potential explanations for this finding. For example, it is possible that the more missing person posters a participant sees, the more likely they will be to later encounter a non-missing person resembling one of them, leading to a higher number of mistaken identifications.

\section{Repeated Exposure to Posters and The Passage of Time}

The present study is the first to demonstrate that seeing missing person posters more than once, when each exposure occurs in quick succession, improves PPM performance. The effect was medium sized. This improvement was predicted as face recognition research has shown that repeated exposure to faces improves later recognition of them (e.g., Roark et al., 2006). Similarly, prospective memory research has shown repeated exposure to cues increases the likelihood of remembering to carry out intentions associated with them when the cues are next encountered (Guynn \& McDaniel, 2007). It is likely that repeated exposure to missing person posters creates a stronger memory of the missing people, improving later PPM performance. Interestingly, it was also found that being exposed to missing person 
posters five times was no more beneficial than being exposed to them three times. This finding is analogous to that observed in advertising research where memory of poster advertisements has been assessed. In a review of that literature, Pechmann and Stewart (1989) showed that repeated exposure to poster advertisements can increase recall of their content, but the memory benefits plateau after six exposures ${ }^{2}$. It is likely that after several exposures to missing person posters/poster advertisements, the memory of them formed is strong enough so that further exposures offer no additional benefit. Finally, the number of times participants saw the missing persons poster did not influence response bias.

Our study is also the first to demonstrate that a delay between seeing missing person posters and later encountering the missing people from them impairs PPM performance. Here the delay lasted an average of $41.29 \mathrm{hr}$ and the impairment was small. This effect was predicted as retrospective memory research shows memory of once-seen faces declines over time (e.g., Deffenbacher et al., 2008) and prospective memory research shows that when the delay between forming a prospective memory intention and encountering the cue to act it is more than a few hr, prospective memory performance can decline (see Martin et al., 2011, for a review and exceptions).

Finally, this study is also the first to show that participants' conservative response bias increases over time, meaning they are more likely to class people as not missing. This effect was small. It is possible that as the participants started to forget what the missing people looked like, they became more conservative in their judgements.

\footnotetext{
${ }^{2}$ Whilst missing person posters and poster advertisements are inherently different, both should aim to make the details presented on them as memorable as possible. Researchers interested in determining ways of making missing person posters more memorable may therefore wish to consider whether any lessons can be learned from advertising researchers, as they have studied this issue for decades (e.g., Craig, Sternthal, \& Leavitt, 1976).
} 


\section{Limitations, Implications, and Future Research}

Laboratory-based PPM studies, such as ours, have low levels of ecological validity, with the procedures used producing higher levels of PPM performance than would be observed in everyday contexts. One reason for this is that in everyday contexts, there is no guarantee members of the public will attend to any missing person posters they see and, if they do, there is also no guarantee they will attend to any missing people from those posters they later encounter. In laboratory-based PPM studies, however, participants are typically required to attend to both. Another reason is that in everyday contexts, members of the public do not necessarily expect to encounter missing people and they are not deliberately put back into the mindset of searching for those people in the moments before encountering one of them. In laboratory-based PPM studies, participants expect to encounter missing people and are reminded to search for them in the moments before encountering them. The low levels of ecological validity do not, however, invalidate our findings. Ecological validity is typically low in memory and law studies, whether examining eyewitness's memory of crimes (e.g., Loftus, Loftus, \& Messo, 1987) or jurors' memory of trial evidence (e.g., Thorley, Baxter, \& Lorek, 2016). The goal of memory and law studies is not to replicate everyday contexts exactly but to examine the impact of key causal variables, in a controlled manner, that are present in everyday contexts and that may influence memory performance in them. The present study did this by controlling and manipulating three factors that may impact upon everyday PPM performance.

Whilst the shortcomings of the current research must be borne in mind, the knowledge generated is useful. For example, it is useful to know that exposing members of the public to a smaller number of posters several times in quick succession may aid PPM performance. If this effect were to be replicated in more ecologically valid research, it may be beneficial for organisations that distribute missing person posters to try and maximise the number of 
exposures that members of the public have to a poster in a short space of time (e.g., by placing several copies of the poster at nearby geographic locations, if this would increase the likelihood of re-exposure). It is, however, important to emphasise that other factors, not considered in this study, may also impact upon the effectiveness of repeatedly exposing members of the public to posters. For example, members of the public may choose to ignore previously seen posters on subsequent exposures, which could nullify any benefits of repeated exposure to the posters. Factors such as this must be considered, and examined in more ecologically valid research, before any policy recommendations are made.

It is also useful to know that PPM performance may decline when the delay between seeing a poster and seeing a missing person is more than $24 \mathrm{hr}$. Again, if this effect were to be replicated in more ecologically valid research, it may be beneficial for organisations that distribute missing person posters to try and ensure members of the public are re-exposed to posters over time to reduce forgetting. As mentioned, however, the role of other factors, such as the amount of attention paid to posters during repeated exposures, would need considering before any policy recommendations are made.

It is also useful to know that members of the public may have a conservative response bias when deciding whether to class a person as missing or not. A conservative response bias has both advantages and disadvantages. An advantage is that members of the public may be less likely to report non-missing people as missing, so police resources are not wasted investigating reports that will not help locate a missing person. A disadvantage, however, is that an actual missing person could be sighted but not reported, meaning an opportunity to locate a missing person is missed. Should this conservative response bias be evident in more ecologically valid research, policy makers could consider whether it is desirable or whether members of the public should be encouraged to report potential sightings of missing people, regardless of their certainty, to avoid missed opportunities. 
Finally, it is important to acknowledge that many other factors, not discussed so far, may also influence PPM performance in everyday contexts. To illustrate this, two will be discussed. The first is the number of missing person pictures there are on a poster. Evidence suggests seeing several different pictures of a missing person on a poster improves PPM performance (Sweeney \& Lampinen, 2012; see also Lampinen et al., 2016, for a related finding). In the present study, participants saw a single picture of a missing person. If multiple pictures appeared on a poster, and participants were repeatedly exposed to these multiple pictures, PPM performance may be even greater than was observed here. Future research examining this possibility is encouraged. The second factor is the relative demographics of the people who are viewing the posters and the missing people depicted on them. Face recognition research suggests people are better are remembering the faces of strangers from ingroups than outgroups, with the ingroups including their own racial group (e.g., Rhodes \& Anastasi, 2012), age group (e.g., Anastasi \& Rhodes, 2006), and gender group (e.g., Wright \& Sladden, 2003). Given that PPM requires face recognition, it is possible that these ingroup biases also impact upon PPM. Across the PPM literature, the relative demographics of participants and missing people have varied but the impact of this on PPM performance is unknown. An example of this variation can be seen in the present study and the study it was partially replicating (Lampinen et al., 2012b, Experiment 1), as both had adult participants but the former involved missing adults and the latter involved missing children. Future studies examining the impact of these relative demographics on PPM performance is also encouraged.

\section{Conclusion}

Globally, thousands of people go missing each day. Members of the public may be asked to assist in locating missing people, with the appeal made via missing person posters placed in high foot-traffic areas. Those distributing the posters hope that members of the 
public who see the posters will later encounter a missing person from one of them, recognise the missing person, and alert authorities of the encounter. It is therefore important to understand the conditions under which this is most likely to occur. The findings from our laboratory-based study suggest this is most likely to occur after seeing four posters (relative to 12), after seeing the posters three to five times in quick succession (relative to once), and when the missing person is encountered shortly after seeing a poster (relative to after a $24 \mathrm{hr}$ delay). When seeing posters once, however, the number of posters seen does not seemingly impact upon PPM performance. Importantly, there were only small-to-moderate declines in performance in the less ideal conditions. Attempts to replicate and extend these findings using more ecologically valid methods are essential before any policy recommendations should be made. 


\section{References}

Anastasi, J. S., \& Rhodes, M. G. (2006). Evidence for an own-age bias in face recognition. North American Journal of Psychology, 8(2), 237-252.

Anderson, F. T., Strube, M. J., \& McDaniel, M. A. (2019). Toward a better understanding of costs in prospective memory: A meta-analytic review. Psychological Bulletin, 145(11), 1053-1081. doi:10.1037/bul0000208

Chance, J. E., \& Goldstein, A. G. (1979). Reliability of face recognition performance. Bulletin of the Psychonomic Society, 14(2), 115-117. doi:10.3758/BF03329416

Cohen, A. L. (2013). Attentional decoupling while pursuing intentions: A form of mind wandering? Frontiers in Psychology, 4, 693. doi:10.3389/fpsyg.2013.00693

Craig, C. S., Sternthal, B., \& Leavitt, C. (1976). Advertising wearout: An experimental analysis. Journal of Marketing Research, 13(4), 365-372. doi:10.2307/3151019

Deffenbacher, K. A., Bornstein, B. H., McGorty, E. K., \& Penrod, S. D. (2008). Forgetting the once-seen face: Estimating the strength of an eyewitness's memory representation. Journal of Experimental Psychology: Applied, 14(2), 139-150. doi:10.1037/1076898X.14.2.139

Einstein, G. O., \& McDaniel, M. A. (1990). Normal aging and prospective memory. Journal of Experimental Psychology: Learning, Memory, and Cognition, 16(4), 717-726. doi:10.1037/0278-7393.16.4.717

Einstein, G. O., \& McDaniel, M. A. (2005). Prospective memory: Multiple retrieval processes. Current Directions in Psychological Science, 14(6), 286-290. doi: 10.1111/j.0963-7214.2005.00382.x

Feng, K., Zhao, X., Liu, J., Cai, Y., Ye, Z., Chen, C., \& Xue, G. (2019). Spaced learning enhances episodic memory by increasing neural pattern similarity across repetitions. 
Journal of Neuroscience, 39(27), 5351-5360. doi:10.1523/JNEUROSCI.274118.2019

Faul, F., Erdfelder, E., Lang, A.-G., \& Buchner, A. (2007). G*Power 3: A flexible statistical power analysis program for the social, behavioral, and biomedical sciences. Behavior Research Methods, 39(2), 175-191. doi:10.3758/BF03193146

Gier, V. S., Kreiner, D. S., \& Hudnell, W. J. (2012). AMBER alerts: are school-type photographs the best choice for identifying missing children?. Journal of Police and Criminal Psychology, 27(1), 9-23. doi:10.1007/s11896-011-9085-z

Goldstein, A. G., \& Chance, J. E. (1971). Visual recognition memory for complex configurations. Perception \& Psychophysics, 9(2), 237-241. doi:10.3758/BF03212641

Guynn, M. J., \& McDaniel, M. A. (2007). Target preexposure eliminates the effect of distraction on event-based prospective memory. Psychonomic Bulletin \& Review, 14(3), 484-488. doi:10.3758/BF03194094

Lampinen, J., Arnal, J. D., Adams, J., Courtney, K., \& Hicks, J. L. (2012a). Forensic age progression and the search for missing children. Psychology, Crime, and Law, 18(4), 405-415. doi:10.1080/1068316X.2010.499873

Lampinen, J.M., Arnal, J.D., \& Hicks, J.L. (2009). Prospective person memory. In M. Kelley (Ed.) Applied Memory. (pp. 167-184). Hauppauge NY: Nova

Lampinen, J. M., Curry, C. R., \& Erickson, W. B. (2016). Prospective person memory: The role of self-efficacy, personal interaction, and multiple images in recognition of wanted persons. Journal of Police and Criminal Psychology, 31(1), 59-70. doi:10.1007/s11896-015-9164-7

Lampinen, J. M., \& Moore, K. N. (2016a). Prospective Person Memory in the Search for Missing Persons. In Morewitz S., \& Sturdy Colls C. (Eds). Handbook of Missing Persons (pp. 145-162). Springer International Publishing. 
Lampinen, J. M., \& Moore, K. N. (2016b). Missing person alerts: does repeated exposure decrease their effectiveness? Journal of Experimental Criminology, 12(4), 587-598. doi:10.1007/s11292-016-9263-1

Lampinen, J. M., Peters, C. S., \& Gier, V. S. (2012b). Power in numbers: The effect of target set size on prospective person memory in an analog missing child scenario. Applied Cognitive Psychology, 26(5), 702-708. doi:10.1002/acp.2848

Loftus, E.F., Loftus, G.R., \& Messo, J. (1987). Some facts about weapon focus. Law and Human Behavior, 11(1), 55-62. doi:10.1007/BF01044839

Lundqvist, D., Flykt, A., \& Öhman, A. (1998). The Karolinska Directed Emotional Faces KDEF, CD ROM from Department of Clinical Neuroscience, Psychology section. Karolinska Institutet, ISBN 91-630-7164-9

Martin, B. A., Brown, N. L., \& Hicks, J. L. (2011). Ongoing task delays affect prospective memory more powerfully than filler task delays. Canadian Journal of Experimental Psychology/Revue Canadienne de Psychologie Expérimentale, 65(1), 48-56. doi:10.1037/a0022872

McDaniel, M. A., \& Einstein, G. O. (2007). Prospective memory: An overview and synthesis of an emerging field. Sage Publications.

Memon, A., Hope, L., \& Bull, R. (2003). Exposure duration: Effects on eyewitness accuracy and confidence. British Journal of Psychology, 94(3), 339-354. doi: $0.1348 / 000712603767876262$

Moore, K. N., \& Lampinen, J. M. (2019). The role of attention and memory in search for missing persons. Journal of Applied Research in Memory and Cognition, 8(2), 189-201. doi:10.1016/j.jarmac.2019.01.005

Moore, K. N., Lampinen, J. M., \& Provenzano, A. C. (2016). The role of temporal and spatial information cues in locating missing persons. Applied Cognitive Psychology, 30(4), 
514-525. doi:10.1002/acp.3242

National Crime Agency (2019). Missing persons data report 2016/2017. London: NCA.

Retrieved from https://www.nationalcrimeagency.gov.uk/who-weare/publications/304-2016-17-ukmpu-data-report-v1/file

National Crime Information Centre (2020). 2019 NCIC missing person and unidentified person statistics. Retrieved from https://www.fbi.gov/file-repository/2019-ncicmissing-person-and-unidentified-person-statistics.pdf/view

Pechmann, C., \& Stewart, D. W. (1989). Advertising repetition: A critical review of wearin and wearout. Current Issues and Research in Advertising, 11(1-2), 285-329.

Podd, J. (1990). The effects of memory load and delay on facial recognition. Applied Cognitive Psychology, 4(1), 47-59. doi:10.1002/acp.2350040105

Rhodes, M. G., \& Anastasi, J. S. (2012). The own-age bias in face recognition: A metaanalytic and theoretical review. Psychological Bulletin, 138(1), 146-174. doi: $10.1037 / \mathrm{a} 0025750$

Roark, D. A., O'Toole, A. J., Abdi, H., \& Barrett, S. E. (2006). Learning the moves: The effect of familiarity and facial motion on person recognition across large changes in viewing format. Perception, 35(6), 761-773. doi:10.1068/p5503

Russo, R., Parkin, A. J., Taylor, S. R., \& Wilks, J. (1998). Revising current two-process accounts of spacing effects in memory. Journal of Experimental Psychology: Learning, Memory, and Cognition, 24(1), 161-172. doi:10.1037//0278-7393.24.1.161

Shapiro, P. N., \& Penrod, S. (1986). Meta-analysis of facial identification studies. Psychological Bulletin, 100(2), 139-156. doi:10.1037/0033-2909.100.2.139

Stanislaw, H. \& Todorov, N. (1999). Calculation of signal detection theory measures. Behavior Research Methods, Instruments, \& Computers, 31(1), 137-149. 
Sweeney, L. N., \& Lampinen, J. M. (2012). The effect of presenting multiple images on prospective and retrospective person memory for missing children. Journal of Applied Research in Memory and Cognition, 1(4), 235-241. doi:10.1016/j.jarmac.2012.08.001

Thorley, C., Baxter, R. E., \& Lorek, J. (2016). The impact of note taking style and note availability at retrieval on mock jurors' recall and recognition of trial information. Memory, 24(4), 560-574. doi:10.1080/09658211.2015.1031250.

Wright, D. B., \& Sladden, B. (2003). An own gender bias and the importance of hair in face recognition. Acta Psychologica, 114(1), 101-114. doi: 10.1016/s0001-6918(03)000520

Xue, G., Mei, L., Chen, C., Lu, Z. L., Poldrack, R., \& Dong, Q. (2011). Spaced learning enhances subsequent recognition memory by reducing neural repetition suppression. Journal of Cognitive Neuroscience, 23(7), 1624-1633. doi:10.1162/jocn.2010.21532

Zhou, H., \& Fishbach, A. (2016). The pitfall of experimenting on the web: How unattended selective attrition leads to surprising (yet false) research conclusions. Journal of Personality and Social Psychology, 111(4), 493-504. doi:10.1037/pspa0000056 